\title{
Ein einfaches Verfahren zur Herstellung anellierter Thiophene*
}

\author{
Christian R. Noe*, Max Knollmüller und Ernst Wagner \\ Institut für Organische Chemie, Technische Universität Wien, A-1060 Wien, \\ Österreich
}

(Eingegangen 5. August 1985. Angenommen 4. September 1985)

\section{A Simple Method for the Synthesis of Fused Thiophenes}

A simple method for the synthesis of fused thiophenes by reaction of $\alpha$ carboxymethyl substituted cyclic ketones with Lawesson-reagent is described. Considerations concerning the reaction mechanism are given.

(Keywords: Lawesson reagent; Mechanism)

\section{Einleitung}

Bei Versuchen zur Thiolactonisierung von Campheressigsäure $\mathbf{1}$ a mit Lawesson-Reagens $2^{1}$ wurde außer dem gewünschten Produkt $\mathbf{4}^{2}$ ein Nebenprodukt beobachtet, welches als das Thiophenderivat 3 a charakterisiert wurde. Ausgehend von diesem Befund wurde versucht, durch Steuerung der Reaktionsbedingungen die Reaktion gänzlich in Richtung der Verbindung 3 a zu lenken. Schließlich gelang die Herstellung von 3 a in guter Ausbeute durch mehrstündiges Kochen mit 2 Moläquivalenten 2 in Toluol (Schema 1; bei Einsatz von einem Moläquivalent 2 oder weniger war 4 das Hauptprodukt). Weder der Methylester von 1 a als Substrat, noch Phosphorpentasulfid als Reagens führten unter vergleichbaren Reaktionsbedingungen $\mathrm{zu} 3$ a.

Dieses Ergebnis erschien uns vor allem vom präparativen Aspekt bemerkenswert, da Thiophenanellierungen in der Regel zu Produkten führen, welche im Thiophenteil substituiert sind, während im vorliegenden Fall sowohl die $\alpha$ - als auch die $\beta$-Position des Thiophenringes unsubstituiert war. Weiters handelte es sich um eine präparativ wenig aufwendige Einstufensynthese aus einem leicht zugänglichen Edukttyp.

* Herrn Prof. Dr. Hans Suschitzky gewidmet. 


\section{Schema 1}

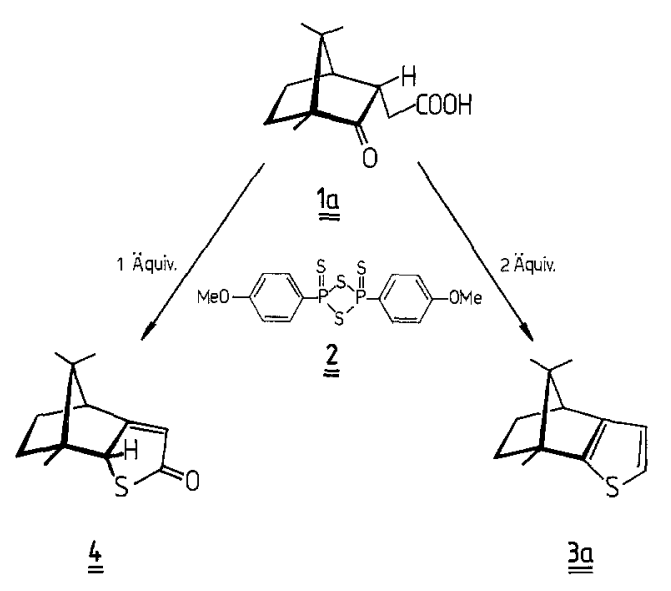

\section{Ergebnisse und Diskussion}

Aus diesen Gründen wurde untersucht, ob das Verfahren verallgemeinerbar ist: dazu wurden die in der Tabelle 1 angeführten cyclischen Ketone $\mathbf{1}$ hergestellt und mit $\mathbf{2}$ in Toluol unter Rückfluß erhitzt. In allen untersuchten Fällen wurde die gewünschte Verbindung $\mathbf{3}$ erhalten. Die Isolierung der Produkte erfolgte in der Regel unter Verwendung einer kurzen Chromatographie-Säule ${ }^{3}$. Obwohl die Reaktionsbedingungen nicht optimiert wurden, waren dic Ausbeuten an 3 zum Teil recht gut.

Während Thiophenanellierungen durch Umsetzungen von Verbindungen vom Typ 1 mit Phosphorsulfiden nicht bekannt sind, ist die reduktive Cyclisierung von $\gamma$-Oxocarbonsäuren ein altbekanntes Verfahren zur Herstellung nicht anellierter Thiophene ${ }^{5}$; es kommen jedoch wesentlich drastischere Reaktionsbedingungen zur Anwendung als im hier beschriebenen Verfahren ${ }^{6}$.

Cyclisierungsversuche mit den Verbindungen 5 ergaben, daß auch nicht anellierte Thiophene 6 beim Umsatz mit 2 in Toluol unter vergleichbar milden Bedingungen erhalten werden können (Tab. 2).

Schließlich war die Reaktion auch vom mechanistischen Aspekt her bemerkenswert, vor allem, da es ja offenbar zu einer reduktiven Entfernung einer Sauerstoff-Funktion aus dem Thiophenring kommt.

Aus diesem Grunde wurden Untersuchungen angestellt, um Hinweise auf den Reaktionsmechanismus zu bekommen. Abgesehen von der Rolle des Lawesson-Reagens als Reduktionsmittel wurde dabei auch das eventuelle Vorliegen einer intramolekularen Hydridverschiebung an einem intermediär gebildeten Zwischenprodukt oder eine Wasserstoff- 
Tabelle 1. Cyclisierung von Ketosäuren 1 zu Thiophenen $\mathbf{3}$

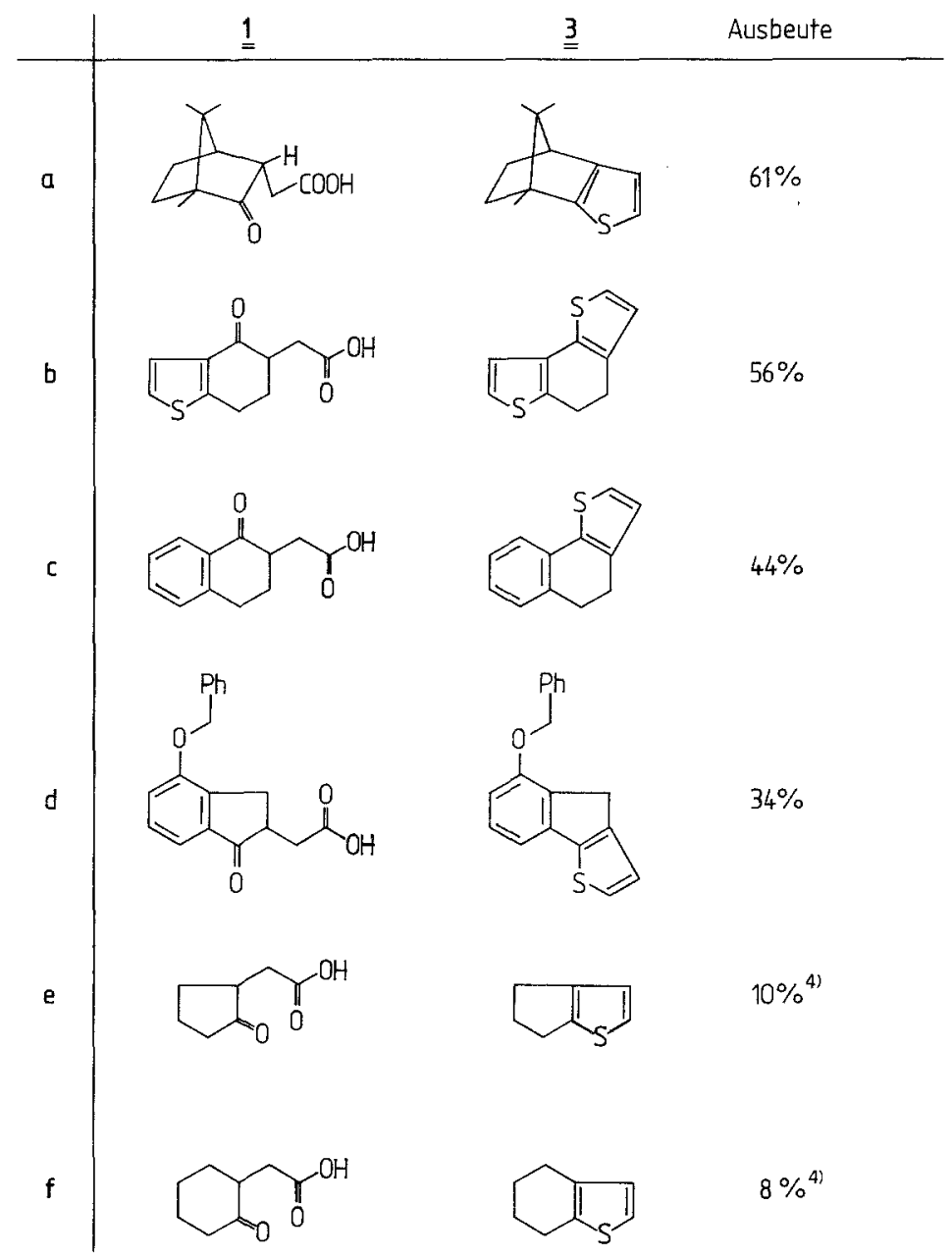

übertragung aus Lösungsmittel-Molekülen (Toluol) für möglich gehalten. So wurde die Verbindung 7 in einer Clemmensen-Reduktion unter Verwendung deuterierter Salzsäure zur Verbindung 8 reduziert, welche an sämtlichen für eine intramolekulare Wasserstoffübertragung relevanten Positionen mit Deuterium substituiert ist. Diese Verbindung wurde den Reaktionsbedingungen der Thiophenanellierung unterworfen und die Produkte $\mathbf{9}$ und $\mathbf{1 0}$ isoliert (Schema 2). 
Tabelle 2. Cyclisierung von Ketosäuren $\mathbf{5}$ zu Thiophenen $\mathbf{6}$

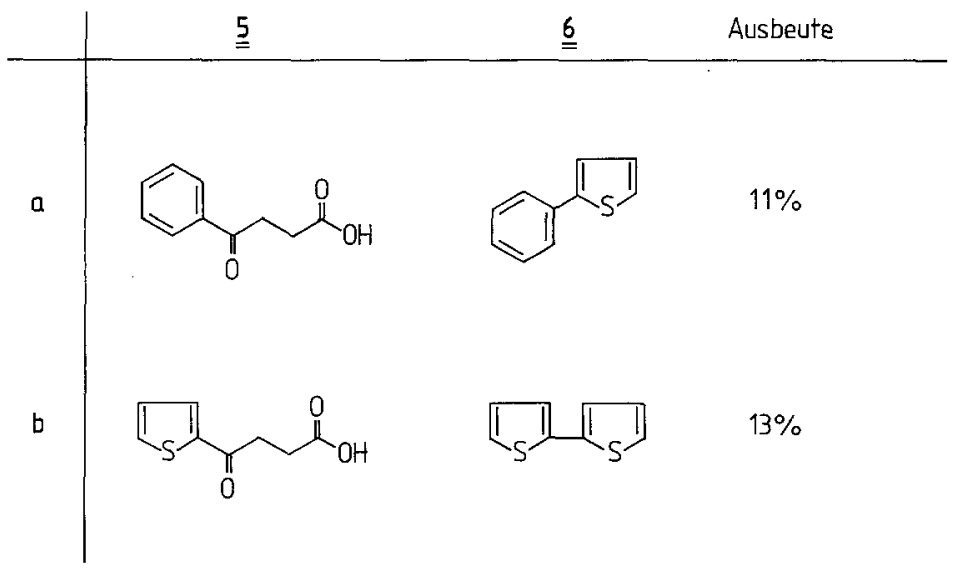

Schema 2

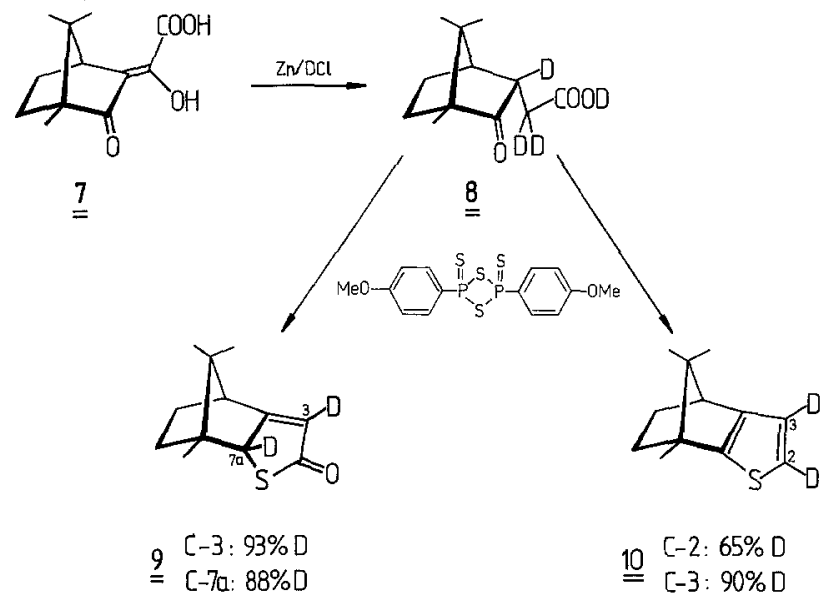

Tatsächlich wurden in den kernresonanzspektroskopischen Untersuchungen beim Thiolacton 9 an der C-3 bzw. C-7 a-Position 93\% bzw. 88\% Deuterium und beim Thiophen 10 an den Kohlenstoffen C-2 bzw. C-3 $65 \%$ bzw. $90 \%$ Deuterium gefunden. Damit konnte also eine Übertragung von Deuterium aus dem Edukt in die Produktmoleküle nachgewiesen und eine Hydridübertragung aus dem Lösungsmittel ausgeschlossen werden.

Die entscheidende $\alpha$-Position (C-2) des Thiophens war wohl mit Deuterium substituiert - wie es im Falle einer intramolekularen Hydrid- 
verschiebung erwartet werden mußte -, wegen der unvollständigen Deuterierung am Kohlenstoff C-2 von $\mathbf{1 0}$ schied jedoch ein streng intramolekularer Mechanismus aus (für die Umsetzung zu 9 kann hingegen eine direkte intramolekulare Wasserstoffübertragung nicht ausgeschlossen werden).

Die Unvollständigkeit der Deuterierung an C-2 von 10 wurde auf möglicherweise über das Lawesson-Reagens eingeschleppte Feuchtigkeit zurückgeführt. Zur Unterstützung dieser Annahme wurden in einem weiteren Experiment vier Äquivalente Deuteriumoxid bei der Umsetzung von nichtdeuterierter Säure 1 a mit 2 zugesetzt. Tatsächlich erfolgte ein Einbau von Deuteriumatomen. Im isolierten Thiophen 10 wurden an den Kohlenstoffen C-2 und C-3 jeweils ungefähr $66 \%$ Deuterium gefunden, was einer statistischen Verteilung entspricht (Verhältnis Moläquivalente Wasser aus Edukt: beigemischtem Deuteriumoxid 1:2).

Die Ergebnisse der Untersuchungen über den Mechanismus der Thiophenanellierung beweisen eine Teilnahme von Deuteriumoxid bzw. Wasser, welches bei der Thiolactonisierung abgespalten wird. Auf Grund des Experiments mit der $\mathrm{D}_{2} \mathrm{O}$-Zumischung kann festgelegt werden, daß der eigentliche Reduktionsmechanismus im wesentlichen über ein mit Wasser bzw. Deuteriumoxid modifiziertes Lawesson-Reagens als Reduktionsmittel verläuft.

\section{Experimenteller Teil}

Schmelzpunkte nach Kofler, nicht korrigiert. Elementaranalysen: Sämtliche Elementaranalysen wurden im Mikroanalytischen Laboratorium am Institut für Physikalische Chemie der Universität Wien unter Leitung von Dr. J. Zak angefertigt. Massenspektren: Massenspektrometer Finnigan 8200; Datensystem Finnigan 8230; Ionisationsenergie $70 \mathrm{eV}$, Beschleunigungsspannung $3 \mathrm{kV}$, Kathodenemissionsstrom $1 \mathrm{~mA}$; Hochauflösung: Auflösung 7000. Sämtliche Massenspektren wurden am Institut für Allgemeine Chemie der Technischen Universität Wien in der Abteilung Dr. J. Varmuza von Ing. J. Dolezal angefertigt. 90-MHz${ }^{1} \mathrm{H}-\mathrm{NMR}$-Spektren und 22.5-MHz- ${ }^{13} \mathrm{C}-\mathrm{NMR}$-Spektren: Jeol FX 90 Q; Chemische Verschiebung in ppm bezogen auf TMS als interner Standard. IR-Spektren: Perkin-Elmer IR 377. Polarimetrie: Perkin-Elmer 241; Messungen in $10 \mathrm{~cm}$ Mikrozelle. Dünnschichtchromatographie (DC): Kieselgel $60 \mathrm{~F}_{254}$, Schichtdicke $0.2 \mathrm{~mm}$ (Merck). Säulenchromatographie (SC): Kieselgel 60, Korngröße 0.040$0.063 \mathrm{~mm}$ (Merck).

Abkürzungen: $E=$ Ether, $P E=$ Petrolether.

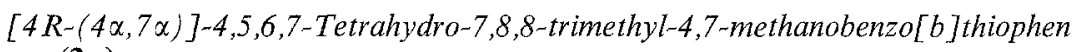

$0.53 \mathrm{~g}(2.50 \mathrm{mmol}) 1 \mathrm{a}^{7}$ und $2.02 \mathrm{~g}(5.00 \mathrm{mmol})$ Lawesson Reagens 2 wurden in $15 \mathrm{ml}$ wasserfreiem Toluol $20 \mathrm{~h}$ unter $\mathrm{N}_{2}$ bei Rückfluß erhitzt. Es wurde zwischen $P E / E(1: 1)$ und wäßriger Natriumhydrogenkarbonatlösung verteilt, mit $P E / E$ extrahiert, gewaschen, getrocknet und eingedampft. Destillation des Rohprodukts bei $120^{\circ} \mathrm{C} / 2$ Torr (Luftbad) ergab $0.294 \mathrm{~g}(61 \%) 3 \mathrm{a}$. Farbloses Öl, Sdp. $70^{\circ} \mathrm{C} / 0.01$ Torr (Luftbad). $[\alpha]_{\mathrm{D}}^{23}=+59^{\circ}(c=1.53$ in $n$-Hexan). IR (KBr): $3060 / 2950(\mathrm{CH})$, 
$1470-1430,830 \mathrm{~cm}^{-1}:{ }^{1} \mathrm{H}-\mathrm{NMR}\left(\mathrm{CDCl}_{3}\right): \delta=7.02 / 6.97 / 6.89 / 6.83(\mathrm{AB} ; 2 \mathrm{H}, 2-$ $\mathrm{H}, 3-\mathrm{H}), 2.87(\mathrm{~d}, J=4 \mathrm{~Hz} ; 1 \mathrm{H}, 4-\mathrm{H}), 2.16-1.54(\mathrm{~m} ; 2 \mathrm{H}$, Aliphaten-H), 1.30 (s; $\left.3 \mathrm{H}, \mathrm{CH}_{3}\right), 1.21-0.93\left(\mathrm{~m} ; 2 \mathrm{H}\right.$, Aliphaten-H), $0.93\left(\mathrm{~s} ; 3 \mathrm{H}, \mathrm{CH}_{3}\right), 0.73(\mathrm{~s} ; 3 \mathrm{H}$, $\left.\mathrm{CH}_{3}\right) \cdot{ }^{13} \mathrm{C}-\mathrm{NMR}\left(\mathrm{CDCl}_{3}\right): \delta=149.5,148.9(2 \mathrm{~s} ; \mathrm{C}-7 \mathrm{a}, \mathrm{C}-3 \mathrm{a}), 122.2,121.3(2 \mathrm{~d} ; \mathrm{C}-$ 3, C-2), 62.0(s; C-8), 54.5 (s; C-7), 51.3 (d;C-4), 34.2(t; C-6), $27.0(\mathrm{t} ; \mathrm{C}-5), 20.0(\mathrm{q}$; $\left.\mathrm{CH}_{3}\right), 19.8\left(\mathrm{q} ; \mathrm{CH}_{3}\right), 12.7\left(\mathrm{q} ; \mathrm{CH}_{3}\right)$.

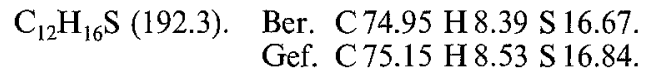

\section{Herstellung der Thiophenderivate 3 (Allgemeine Arbeitsvorschrift)}

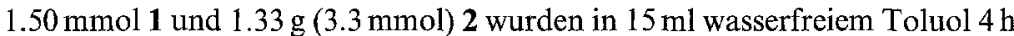
unter $\mathrm{N}_{2}$ bei Rückfluß erhitzt. Es wurde zwischen Wasser und $P E / E(2 / 1)$ verteilt und mit $P E / E$ extrahiert. Die vereinigte organische Phase wurde mit Natriumhydrogenkarbonatlösung gewaschen, getrocknet und eingedampft. Aus dem Rückstand wurde das Produkt 3 durch Säulenchromatographie (Kieselgel, Eluens: $P E$ ) isoliert.

\section{4,5,6,7-Tetrahydro-4-oxobenzo[b]thiophen-5-essigsäure (1 b)}

$\mathrm{Zu}$ einer Suspension von $4.0 \mathrm{~g}$ (92 mmol) 55\% Natriumhydrid in Paraffinöl und $16.6 \mathrm{~g}(184 \mathrm{mmol})$ Dimethylcarbonat in $60 \mathrm{ml}$ wasserfreiem Benzol wurden bei $60^{\circ} \mathrm{C}$ langsam eine Lösung von $7.0 \mathrm{~g}(46 \mathrm{mmol}) 6,7$-Dihydrobenzo[b]thiophen$4(5 H)$-on ${ }^{8}$ zugetropft. Nach $3 \mathrm{~h}$ bei Rückflußtemp. wurde auf $0^{\circ} \mathrm{C}$ gekühlt und langsam $10 \mathrm{ml}$ Eisessig zugegeben. Das Reaktionsgemisch wurde zwischen $5 \%$ Essigsäure und $P E / E(1: 1)$ verteilt, die organische Phase abgetrennt und die wäßrige noch zweimal mit $P E / E$ extrahiert. Die vereinigten organischen Phasen wurden mit Natriumhydrogencarbonatlösung gewaschen, mit Natriumsulfat getrocknet und im Vakuum eingedampft. Das erhaltene Zwischenprodukt ( $9.57 \mathrm{~g})$ wurde in $40 \mathrm{ml}$ wasserfreiem $D M F$ gelöst und mit $2.0 \mathrm{~g} \mathrm{(46} \mathrm{mmol}) 55 \%$ Natriumhydrid versetzt. Es wurde $1 \mathrm{~h}$ bei $40^{\circ} \mathrm{C}$ gerührt, bei Raumtemp. $7.7 \mathrm{~g} \mathrm{(46} \mathrm{mmol)}$ Bromessigsäureethylester zugetropft und das Reaktionsgemisch $2 \mathrm{~h}$ auf $70^{\circ} \mathrm{C}$ gehalten. Das $D M F$ wurde im Vakuum entfernt und der Rückstand zwischen Wasser und $P E / E(1: 1)$ verteilt. Die organische Phase wurde gewaschen, getrocknet und im Vakuum eingedampft. Der Rückstand $(12.67 \mathrm{~g})$ wurde in $70 \mathrm{ml}$ $70 \%$ wäßrigem Methanol gelöst und bei Siedehitze eine Lösung von $8 \mathrm{~g}$ Kaliumhydroxid in $40 \mathrm{ml} \mathrm{70 \%}$ Ethanol langsam zugetropft. Nach $3 \mathrm{~h}$ wurde im Vakuum eingeengt, mit $150 \mathrm{ml}$ Wasser verdünnt und mit $E$ gewaschen. Die wäßrige Phase wurde angesäuert und mit Dichlormethan extrahiert, die organische Phase getrocknet und eingedampft. Umkristallisieren des Rohproduktes ergab $6.3 \mathrm{~g} \mathrm{(65 \% )} \mathrm{farblose} \mathrm{Kristalle,} \mathrm{Schmp.} 119-120^{\circ} \mathrm{C}$.

${ }^{\mathrm{I}} \mathrm{H}-\mathrm{NMR}\left(\mathrm{CDCl}_{3}\right): \delta=9.0$ (br. s; $\left.1 \mathrm{H}, \mathrm{OH}\right), 7.39 / 7.34(\mathrm{AB} ; 1 \mathrm{H}, 2-\mathrm{H})$, 7.09/7.02 (AB; $1 \mathrm{H}, 3-\mathrm{H}), 3.3-1.7\left(\mathrm{~m} ; 7 \mathrm{H}\right.$, Aliphaten-H). ${ }^{13} \mathrm{C}-\mathrm{NMR}\left(\mathrm{CDCl}_{3}\right)$ : $\delta=192.8 / 177.7$ (2s; C-4, COO), 155.3/136.1 (2s; C-7a, C-3a), 124.6/123.3 (2d; C-2, C-3), 43.1 (d; C-5), 34.0/30.1/24.9 (3t; C-6, C-7, C- $\alpha$ ).

$\mathrm{C}_{10} \mathrm{H}_{10} \mathrm{O}_{3} \mathrm{~S}(210.25)$ : Ber. C 57.13 H 4.79. Gef. C $57.33 \mathrm{H} 4.86$.

\section{4,5-Dihydrobenzo [1,2-b: 3,4-b']dithiophen (3 b)}

Aus $0.30 \mathrm{~g}(1.43 \mathrm{mmol}) \mathbf{1 b}$ nach der allgemeinen Arbeitsvorschrift wurden $153 \mathrm{mg}(56 \%) \mathbf{3} \mathbf{b}^{9}$ erhalten.

${ }^{1} \mathrm{H}-\mathrm{NMR}\left(\mathrm{CDCl}_{3}\right): \delta=7.07 / 7.02(\mathrm{AB} ; 2 \mathrm{H}, 2-\mathrm{H}, 7-\mathrm{H}), 6.91 / 6.85(\mathrm{AB} ; 2 \mathrm{H}$, $3-\mathrm{H}, 8-\mathrm{H}), 3.0\left(\mathrm{~s} ; 4 \mathrm{H}, \mathrm{CH}_{2}-\mathrm{CH}_{2}\right)$. 


\section{4,5-Dihydronaphtho[1,2-b]thiophen (3c)}

Aus $1.00 \mathrm{~g}(4.90 \mathrm{mmol}) \mathbf{1} \mathrm{c}^{10}$ wurden nach der allgemeinen Arbeitsvorschrift $0.40 \mathrm{~g}(44 \%) \mathbf{3} \mathbf{c}^{11}$ erhalten.

${ }^{1} \mathrm{H}-\mathrm{NMR}\left(\mathrm{CDCl}_{3}\right): \delta=7.4-7.1(\mathrm{~m} ; 5 \mathrm{H}$, Aromaten-H), 6.95/6.87 (AB; $1 \mathrm{H}$, $3-\mathrm{H}), 3.0-2.8\left(\mathrm{~m} ; 4 \mathrm{H}, \mathrm{CH}_{2}-\mathrm{CH}_{2}\right)$.

2,3-Dihydro-1-oxo-4-(phenylmethoxy)-1 H-inden-2-essigsäure (1 d)

Hergestellt analog der Vorschrift für $\mathbf{1}$ b.

${ }^{1} \mathrm{H}-\mathrm{NMR}\left(\mathrm{CDCl}_{3}\right): \delta=11.4$ (br. s; $\left.1 \mathrm{H}, \mathrm{OH}\right), 7.6-7.0(\mathrm{~m} ; 8 \mathrm{H}$, Aromaten-H), $5.12\left(\mathrm{~s} ; 2 \mathrm{H}, \mathrm{OCH}_{2} P h\right), 3.6-2.2(\mathrm{~m} ; 5 \mathrm{H}$, Aliphaten- $\mathrm{H})$.

\section{5-Phenylmethoxy-4H-indenothiophen (3 d)}

Aus $0.25 \mathrm{~g}(0.85 \mathrm{mmol}) \mathbf{1} \mathbf{d}$ wurden nach der allgemeinen Arbeitsvorschrift $82 \mathrm{mg}(34 \%) 3 \mathrm{~d}$ erhalten.

${ }^{1} \mathrm{H}-\mathrm{NMR}\left(\mathrm{CDCl}_{3}\right): \delta=7.60-6.75(\mathrm{~m} ; 10 \mathrm{H}$, Aromaten-H), $5.20(\mathrm{~s} ; 2 \mathrm{H}, P h-$ $\left.\mathrm{CH}_{2}-\mathrm{O}\right), 3.70(\mathrm{~s} ; 2 \mathrm{H}, 4-\mathrm{H})$.

\section{5,6-Dihydro-4 H-cyclopentathiophen (3 e)}

Aus $1.00 \mathrm{~g}(7.04 \mathrm{mmol}) 1 \mathrm{e}^{12}$ wurden nach der allgemeinen Arbeitsvorschrift $90 \mathrm{mg}(10 \%) 3 \mathrm{e}^{13}$ erhalten.

${ }^{1} \mathrm{H}-\mathrm{NMR}\left(\mathrm{CDCl}_{3}\right): \delta=7.16 / 7.11(\mathrm{AB} ; 1 \mathrm{H}, 2-\mathrm{H}), 6.80 / 6.76(\mathrm{AB} ; 1 \mathrm{H}, 3-\mathrm{H})$, $3.0-2.2(\mathrm{~m} ; 6 \mathrm{H}$, Aliphaten-H).

\section{4,5,6,7-Tetrahydrobenzo[b]thiophen (3f )}

Aus $2.67 \mathrm{~g}$ (17.1 mmol) $1 \mathrm{f}^{14}$ wurden nach der allgemeinen Arbeitsvorschrift $190 \mathrm{mg}(8 \%) 3 \mathbf{f}^{15}$ erhalten.

${ }^{1} \mathrm{H}-\mathrm{NMR}\left(\mathrm{CDCl}_{3}\right): \delta=6.93 / 6.87(\mathrm{AB} ; 1 \mathrm{H}, 2-\mathrm{H}), 6.65 / 6.59(\mathrm{AB} ; 1 \mathrm{H}, 3-\mathrm{H})$, $2.8-2.4\left(\mathrm{~m} ; 4 \mathrm{H}, 4-\mathrm{CH}_{2}, 7-\mathrm{CH}_{2}\right), 2.0-1.5\left(\mathrm{~m} ; 4 \mathrm{H}, 5-\mathrm{CH}_{2}, 6-\mathrm{CH}_{2}\right)$.

\section{2-Phenylthiophen (6a)}

Aus $0.44 \mathrm{~g}(2.50 \mathrm{mmol}) 5 \mathrm{a}^{16}$ wurden nach der allgemeinen Arbeitsvorschrift (6h bei Rückflußtemperatur) $45 \mathrm{mg}(11 \%) 6 \mathrm{a}^{17}$ erhalten.

${ }^{1} \mathrm{H}-\mathrm{NMR}\left(\mathrm{CDCl}_{3}\right): \delta=7.22-6.93(\mathrm{~m} ; 6 \mathrm{H})$.

\section{$2,2^{\prime}-$ Bithiophen $(\mathbf{6} \mathbf{b})$}

Aus $0.46 \mathrm{~g}(2.50 \mathrm{mmol}) \mathbf{5} \mathbf{b}^{18}$ wurden nach der allgemeinen Arbeitsvorschrift ( $8 \mathrm{~h}$ bei Rückflußtemperatur) $45 \mathrm{mg}(13 \%) \mathbf{6 b}^{19}$ erhalten.

${ }^{1} \mathrm{H}-\mathrm{NMR}\left(\mathrm{CDCl}_{3}\right): \delta=6.9-7.3(\mathrm{~m} ; 6 \mathrm{H}) .{ }^{13} \mathrm{C}-\mathrm{NMR}\left(\mathrm{CDCl}_{3}\right): \delta=137.9(\mathrm{~s})$, 127.4 (d), 124.0 (d), 123.5 (d).

\section{(1R-endo)-4,7,7-Trimethyl-3-oxobicyclo[2.2.1] ]heptan-2-essig-2, $\alpha, \alpha-d_{3}-$ säure $-d$ \\ (Tetradeuterocampheressigsäure) (8)}

$8 \mathrm{~g}$ Zinkstaub und $0.8 \mathrm{~g}$ Quecksilber(II)chlorid wurden mit $10 \mathrm{ml}$ Deuteriumoxid und $1 \mathrm{ml}$ einer $20 \%$ Lösung von Deuteriumchlorid in Deuteriumoxid ca. $5 \mathrm{~min}$ bei Raumtemp. gerührt. Die Lösung wurde abdekantiert, $27 \mathrm{ml}$ einer $20 \%$ Deuteriumchloridlösung und $2.4 \mathrm{~g}$ D-Campheroxalsäure $7^{20}$ zugegeben, und bei Rückfluß erhitzt. Nach $30 \mathrm{~h}$ wurde die Reaktionslösung mit $3 \mathrm{ml}$ einer $20 \%$ Deuteriumchlorid-Lösung angesäuert und mit wasserfreiem Dichlormethan vier- 
mal extrahiert. Die vereinigten organischen Phasen wurden über wasserfreiem Natriumsulfat getrocknet, filtriert und im Vakuum eingedampft. Ausbeute $1.14 \mathrm{~g}$ $(50 \%)$ 8. Farblose Kristalle, Schmp. $74-79^{\circ} \mathrm{C}$ (aus $P E / E$ ).

${ }^{\prime} \mathrm{H}-\mathrm{NMR}\left(\mathrm{CDCl}_{3}\right): \delta=2.22(\mathrm{~d}, J=3.2 \mathrm{~Hz} ; 1 \mathrm{H}, 1-\mathrm{H}), 1.90-1.21(\mathrm{~m} ; 4 \mathrm{H}$, Aliphaten-H), $1.03\left(\mathrm{~s} ; 3 \mathrm{H}, \mathrm{CH}_{3}\right), 0.90\left(\mathrm{~s} ; 6 \mathrm{H}, 2 \mathrm{CH}_{3}\right)$; die quantitative Auswertung des Signals bei $11.3(\mathrm{~s} ; \mathrm{COOH})$ zeigte einen Deuterierungsgrad an der Carboxylgruppe von ca. $85 \%$.

$$
\mathrm{C}_{12} \mathrm{H}_{14} \mathrm{D}_{4} \mathrm{O}_{3} \mathrm{MS} \text { (Hochauflösung): Ber. 214.1506. Gef. 214.1496+0.0011. }
$$

Zum Zinkamalgam-Rückstand der Aufarbeitung wurden $40 \mathrm{ml} 20 \%$ Salzsäure zugegeben, mit der Deuteriumoxid/Deuteriumchlorid-Phase der Aufarbeitung vereinigt und die Lösung mit Dichlormethan extrahiert, mit wenig verdünnter Salzsäure gewaschen, getrocknet und im Vakuum eingedampft. Ausbeute $0.67 \mathrm{~g}$ $(29 \%) 8$ a ; laut ${ }^{1} \mathrm{H}$-NMR Spektrum $(11.5, \mathrm{~s}, 1 \mathrm{H})$ überwiegend trideuterierte Campheressigsäure.

$$
\mathrm{C}_{12} \mathrm{H}_{15} \mathrm{D}_{3} \mathrm{O}_{3} \text { MS (Hochauflösung): Ber. 213.1444. Gef. 213.1447+0.0011. }
$$

\section{Umsetzung von deuterierter Campheressigsäure $\mathbf{8}$ mit 2}

$0.36 \mathrm{~g}$ (1.68 mmol) 8 wurden mit $0.8 \mathrm{~g}(1.98 \mathrm{mmol}) 2$ nach der allgemeinen Arbeitsvorschrift ( $90 \mathrm{~min}$ bei Rückflußtemp.) umgesetzt und säulenchromatographisch die Produkte 9 und 10 isoliert.

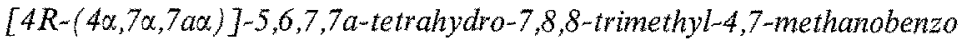 \\ [b] thiophen-3,7a-d $d_{2}-2(4 H) \sim$ on (9)}

Das in obiger Reaktion isolierte Thiolacton stellte hauptsächlich das Dideutero-Analogon von 4 dar; aus dem Protonenkernresonanzspektrum konnte ein Deuterierungsgrad von $93 \%$ Deuterium an $\mathrm{C}-3$ sowie $88 \%$ an $\mathrm{C}-7$ a ermittelt werden.

${ }^{\prime} \mathrm{H}-\mathrm{NMR}\left(\mathrm{CDCl}_{3}\right): \delta=6.02(\mathrm{~s} ; 0.07 \mathrm{H}, 3-\mathrm{H}), 4.73(\mathrm{~s} ; 0.12 \mathrm{H}, 7 \mathrm{a}-\mathrm{H}), 3.03(\mathrm{~d}$, $J=4 \mathrm{~Hz} ; 1 \mathrm{H}, 4-\mathrm{H}), 2.22-1.14\left(\mathrm{~m} ; 4 \mathrm{H}\right.$, Aliphaten-H), $1.01\left(\mathrm{~s} ; 6 \mathrm{H}, 2 \mathrm{CH}_{3}\right), 0.98$ (s; $3 \mathrm{H}, \mathrm{CH}_{3}$ ).

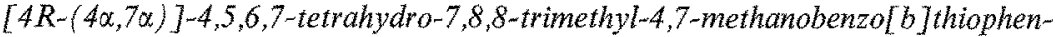 $2,3-d_{2}(\mathbf{1 0})$}

Das aus obiger Reaktion isolierte Thiophen wies einen Deuterierungsgrad von $65 \%$ an $\mathrm{C}-2$ und $90 \%$ an $\mathrm{C}-3$ auf, wie aus dem ${ }^{1} \mathrm{H}-\mathrm{NMR}$ ermittelt werden konnte.

${ }^{1} \mathrm{H}-\mathrm{NMR}\left(\mathrm{CDCl}_{3}\right): \delta=6.98(\mathrm{~s} ; 0.35 \mathrm{H}, 2-\mathrm{H}), 6.87(\mathrm{~s}$, dabei kleine Signalanteile $\delta=6.89 / 6.84) ; 0.10 \mathrm{H}, 3-\mathrm{H}), 2.89(\mathrm{~d}, J=4 \mathrm{~Hz} ; 1 \mathrm{H}, 4-\mathrm{H}), 2.15-1.60(\mathrm{~m} ; 2 \mathrm{H}$, Aliphaten- $\mathrm{H}), 1.30\left(\mathrm{~s} ; 3 \mathrm{H}, \mathrm{CH}_{3}\right), 1.21-0.93(\mathrm{~m} ; 2 \mathrm{H}$, Aliphaten- $\mathrm{H}), 0.93(\mathrm{~s} ; 3 \mathrm{H}$, $\left.\mathrm{CH}_{3}\right), 0.73\left(\mathrm{~s} ; 3 \mathrm{H}, \mathrm{CH}_{3}\right)$.

\section{Umsetzung von $\mathbf{1}$ a mit $\mathbf{2}$ bei Anwesenheit von Deuteriumoxid}

$500 \mathrm{mg}(2.38 \mathrm{mmol}) 1$ a wurden mit $1.70 \mathrm{~g}(4.2 \mathrm{mmol}) 2$ und $200 \mathrm{mg}(10 \mathrm{mmol})$ Deuteriumoxid in $10 \mathrm{ml}$ Toluol $4 \mathrm{~h}$ bei RückfluB erhitzt. Dünnschichtchromatographische Verfolgung der Reaktion zeigte, daß der Zusatz von Deuteriumoxid die Umsetzung zum Thiophenprodukt erheblich verlangsamte und auch die Ausbeute (nach Aufarbeitung analog der allgemeinen Arbeitsvorschrift) verringerte; $100 \mathrm{mg}(22 \%) 10$, Deuterierung an $\mathrm{C}-2$ und $\mathrm{C}-3$ je ca. $65 \%$ laut Protonenkernresonanzspektrum. 


\section{Literatur und Anmerkungen}

1 a) Pedersen B. S., Scheibye S., Nilsson N. H., Lawesson $S . \sim O$. , Bull. Soc. Chim. Belg. 87, 223 (1978). b) Scheibye S., Pedersen B. S., Lawesson S.-O., Ibid. 87, 229 (1978). c) Pedersen B. S., Scheibye S., Clausen K., Lawesson S.-O., Ibid. 87, 293 (1978). d) Scheibye S., Kristensen J., Lawesson S.-O., Tetrahedron 35, 1339 (1979). e) Pedersen B. S., Lawesson S. O., ibid. 35, 2433 (1979).

${ }^{2}$ Noe C. R., Knollmüller M., Wagner E., Völlenkle H., Chem. Ber. 118, 3299 (1985).

3 Auch destillative Isolierung war teilweise möglich (z. B. für 3 a).

${ }^{4}$ Die Reaktionen wurden nicht optimiert, die schlechten Ausbeuten wurden hauptsächlich auf die Flïchtigkeit der Substanzen bei der Abtrennung des Toluols zurückgeführt.

${ }^{5}$ Gronowitz S., Recent Advances in the Chemistry of Thiophenes. In: Advan. Heterocyclic Chem. (Katritzky A. R., Hrsg.), 1, 1-124. New York, N.Y.: Academic Press. 1963. Hirao 1., Yakugaku Zasshi 73, 1023 (1953); Chem. Abstr. 48, 10723.

${ }^{6}$ Reaktionsbedingungen: Erhitzen mit $\mathrm{P}_{2} \mathrm{~S}_{3}$ im Gemisch mit Sand auf 210 $260^{\circ} \mathrm{C}$.

7 Noe C. R., Chem. Ber. 115, 1576 (1982).

${ }^{8}$ Spitz W., Hammer H., D. B.P. 1.108.208 (U 120) (4. 3. 1959).

9 Ricci A., Balucani D., Rossi C., Croisy A., Boll. Sci. Fac. Chim. Ind. Bologna 27, 279 (1969).

10 Severin T., Poehlmann H., Chem. Ber. 111, 1564 (1978).

11 Clarke K., Gregory D. N., Scrowston R. M., J. Chem. Soc. Perkin Trans. I, 1977, 63.

12 Khorgami M. H., Quart. Bull. Fac. Sci. Teheran Univ. 1971, 19; Chem. Abstr. $75,63518 \mathrm{u}$

13 MacDowell D. W. H., Patrick T. B., Frame B. K., Ellison D. L., J. Org. Chem. 32, 1226 (1967).

14 Gula M. J., Spencer T. A., J. Org. Chem. 45, 805 (1980).

15 Cagniant P., Kirsch G., C.R. Hebd. Seances Acad. Sci., Ser, C 281, 35 (1975).

16 Fadnavis N. W., Bagavant G., Indian J. Chem., Sect. B 17 B, 518 (1979).

17 Sotoyama T., Hara S., Suzuki A., Bull. Chem. Soc. Jpn. 52, 1865 (1979).

18 Pitarch L., Coronas R., Mallol J., Eur. J. Med. Chem.-Chim. Ther. 9, 644 (1974).

19 Zembayashi M., Tamao K., Yoshida J. I., Kumada M., Tetrahedron Lett. 1977, 4089.

${ }^{20}$ Langenbeck $W .$, Triem G., Chem. Ber. 69, 248 (1936). 